\title{
Healing After Simple Gingivectomy
}

\author{
BY SIGURD P. RAMFJORD, $\%$ L.D.s., PH.D., AND \\ EMMETT R. COSTICH,** D.D.S., PH.D., ANN ARBOR, MICHIGAN
}

$\mathrm{T}$

HE purpose of this investigation was to study the periodontal reaction to simple gingivectomy which represents the most common modality of periodontal surgery.

A few reports on healing following gingivectomy can be found in the literature. ${ }^{1-5}$ Only one investigation ${ }^{1,3}$ included block sections of teeth and alveolar bone with the periodontal specimens, and in that instance the gingivectomy had been done to the alveolar crest. No documented reports could be found on the postsurgical relationship between the tooth and the healing tissues in humans The experimental investigations in animals ${ }^{\text {i-12 }}$ have not paralleled the conditions of surgery and postoperative care received by patients. Deliberate notches have been made on the surfaces of the animal teeth at the level of the surgery, and there has been lack of surgical dressings, or dressings have been left on the wounds longer than is customary for humans.

With the current interest in refinement of periodontal surgery it seems basic to investigate the reaction of the periodontal tissues in humans to the simplest and most common form of gingivoplasty and gingivectomy, namely, the removal of the gingival tissues to the bottom of the gingival crevice for the purpose of establishing a physiologic gingival sulcus and contour.

MATERIAL

Ten postgingivectomy specimens from nine immediate denture patients at the University of Michigan School of Dentistry were obtained for the histologic part of this study. Clinical measurements and other recordings from the same patients provided the clinical data. The patients were in good health and varied in age from 27 to 48 years. Records with roentgenograms and intraoral pictures were available. At the time of insertion of the immediate dentures, block sections of the experimental anterior teeth with adjacent buccal soft tissues and bone to the apical half of the root were removed. The periodontal health varied from clinically normal gingiva to moderately advanced periodontitis.

\footnotetext{
*The University of Michigan School of Dentistry, Ann Arbor, Michigan.
}

* The University of Kentucky College of Dentistry, Lexington, Kentucky.

Page 5/401 


\section{METHOD}

A standard record providing detailed information with regards to periodontal status and functional relations of the experimental teeth was kept. Prior to the gingivectomy, a horizontal notch was made into dentin on the labial surface of the experimental tooth. A diamond stone was used to make the notch about two millimeters incisally to the gingival margin. Using cotton pliers, a Boley gauge and a blunted \#2 silver point of the type used for root canal therapy, measurements were made from the gingival border of the notch to the free gingival margin and from the notch to the bottom of the gingival crevice or pocket (see Table 1).

A simple gingivectomy or gingivoplasty was then performed with a new BardParker \#1 $2^{\mathrm{B}}$ blade. An attempt was made to provide proper bevel and to remove the gingival tissues incisally to the bottom of the clinically measured gingival crevice. The gingival excision was carried into the interproximal space and over to the adjacent teeth. The surgically exposed tooth surface was planed in a routine manner with periodontal files and curettes. This, of course, included removal of any subgingival calculus present at the time of the surgery (Fig. 1).

The distance from the notch to the soft tissue attachment at the completion of the surgery was then measured and a Wondrpak dressing placed on the wound. If the specimen was not removed within six to seven days, the surgical dressing was changed following debridement of the wound with one and a half per cent hydrogen peroxide in warm water. All presurgical measurements, gingivectomy, and immediate postsurgical measurements were done by the same person (S. R.).

A clinical examination duplicating the pregingivectomy procedures was done before the specimen was removed. This included measurements from the notch to the new free gingival margin and from the notch to the bottom of the crevice (included in Table 1 as posthealing measurements). New intraoral photographs also were taken. These recordings were all made by the other investigator (E. C.).

The specimens then were removed by a modification of the technic described by Kohler and Ramfjord. ${ }^{13}$ Since this new technic has worked extremely well over the past two years for removal of a considerable number of specimens, a detailed description and discussion is included.

The technic for removal of block specimens described by Kohler and Ramfjord ${ }^{13}$ provided adequate specimens, but the time required for complete healing was long, and the immediate postsurgical result did not appear satisfactory. Although this surgical defect eventually healed completely without any residual distortion or mutilation of the alveolar ridge, the present technic has improved the type of specimen, the speed of operation, and provided a simple wound closure.

The various steps for the present technic for removal of specimens are illustrated in Fig. 2. Drawing 1 in Fig. 2 is for the purpose of orientation, showing the left maxillary lateral incisor with the labial notch to be used as a landmark for the clinical and histological measurements. The free gingival margin and the apical boundary of the attached gingiva are indicated. In Drawing 2 of Fig. 2, the dotted line indicates the incision of the mucosa. The incisions are started at point $F$ and carried to $\mathrm{B}$ and to $\mathrm{C}$ in order to prevent distortion while making the cuts. The assistant and the operator must be extremely careful to avoid injuring the specimen with instruments or sponges. A second pass of the knife in the incisions FB and FC is advisable to be sure the periosteum has been incised. A gingival crevice incision is then made from $C$ to $D$ and carried distally and superiorly from the canine tooth through the apical level of the attached gingiva at E. Another crevicular incision is made from $B$ to $A$. The mucoperiosteal flap is reflected beginning at points $\mathrm{B}$ and $\mathrm{C}$ and carried 


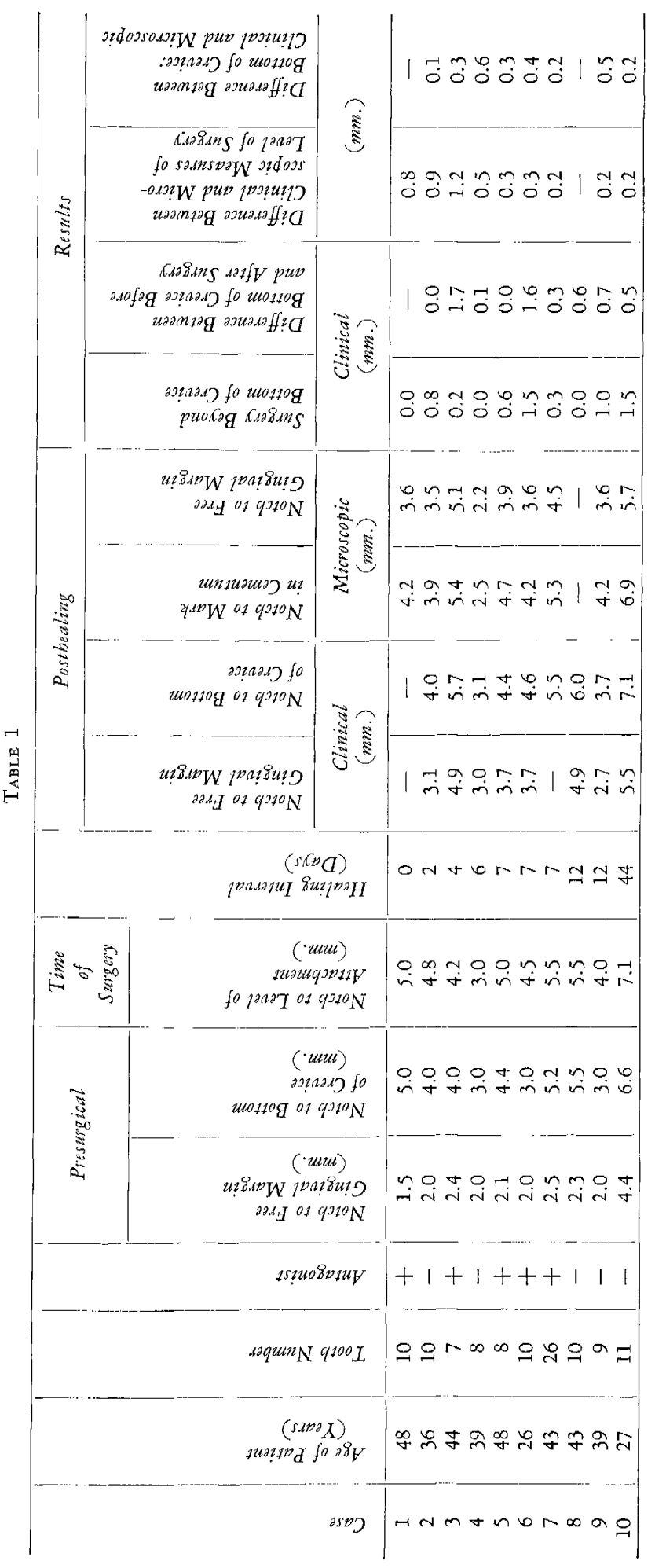


away from the incision lines FB and FC. The flap is reflected high so that the bone is exposed well around the specimen and above the level of the apices of the adjacent teeth. The reflected flap is shown in

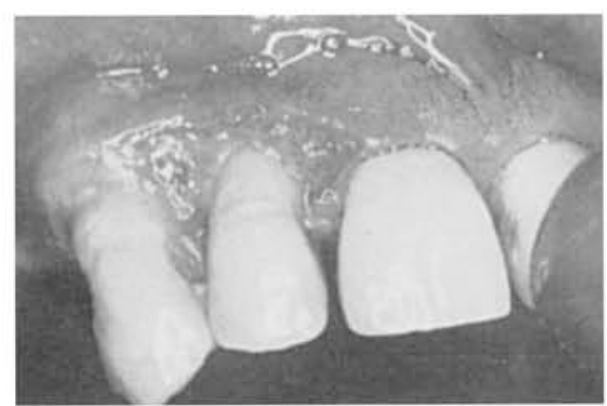

Fig. 1. Clinical pieture of Case 3 four days after gingivectomy. Typical incision for lateral incisor.

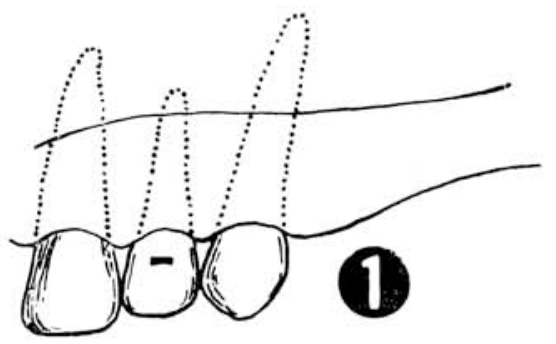

Drawing 3, Fig. 2. The heavy lines along the specimen tooth represent a bur cut into the bone and a connecting cut at the apex of the tooth. These bur cuts are extended through the bone and to the lateral surfaces of the root using a \#4 carbide bur. The cut at the level of the apex is made deep so that the apex can be fractured by inserting a straight elevator in the groove and twisting the elevator quickly. The specimen, including the tooth, is then removed by elevating the root from the socket, working from the apical end. Forceps are not used. The crown is held by the fingers to prevent it from falling when the specimen is loosened. The specimen is immediately placed in fixative. The remaining teeth are extracted and the periosteum is severed with
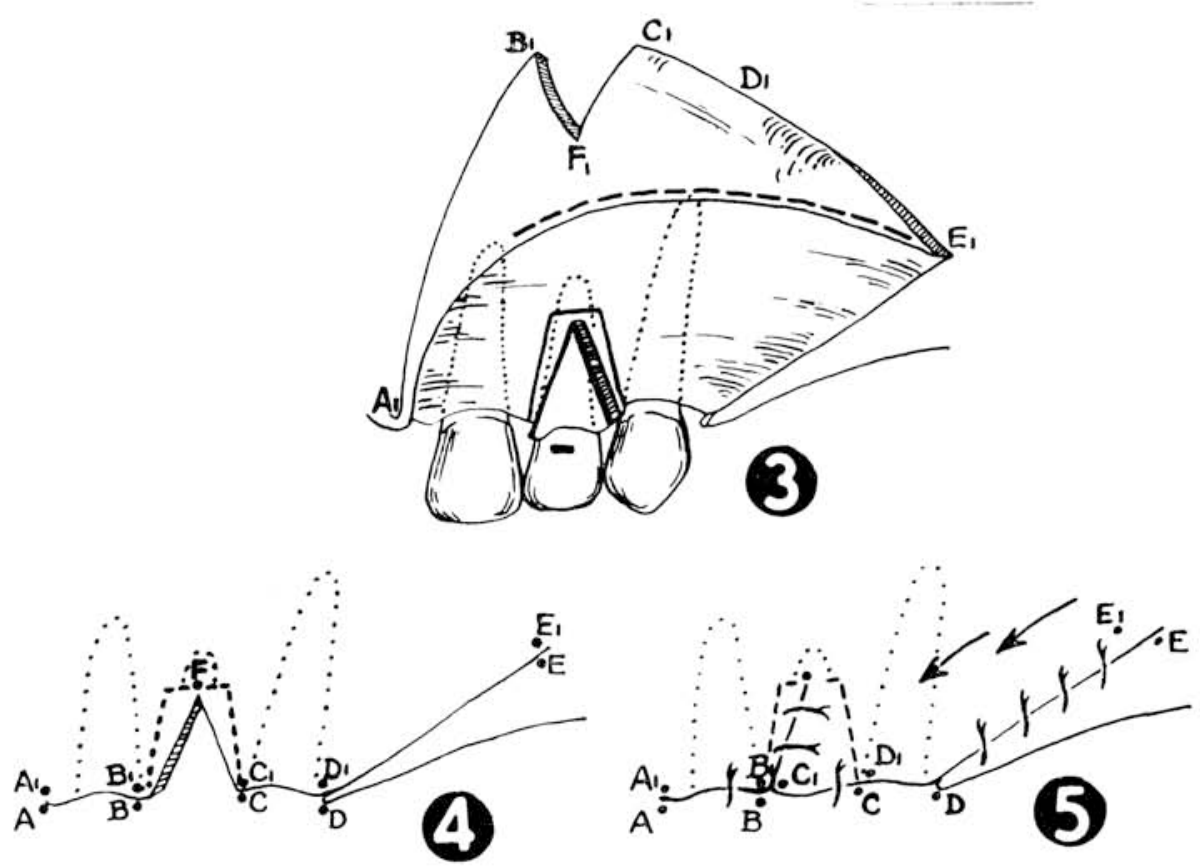

Fig. 2. Drawings illustrating removal of the specimens. 
a sharp shallow cut at the level of the periosteal reflection from the bone. The dotted line in Drawing 3, Fig. 2, indicates where this incision is made. The cut should go through only the periosteum and not through the full thickness of the flap.

Drawing 4, Fig. 2 shows the flap returned to place with the notched defect where the specimen was removed. Drawing 5, Fig. 2, illustrates the closure of the wound. The vertical closure is shifted toward the midline because the periosteum is not cut as far mesially as it is cut laterally. This minimizes displacement of the labial frenum. The letters indicating the points on the drawing have similar letters with subscripts to indicate how the flap shifts. The distance $\mathrm{E}$ to $\mathrm{E}_{1}$ is small because there is very little stretching of the mucosa in this area. The distance $D$ to $D_{1}$ is larger because there is a greater length of flap available to stretch, and an attempt is made to gain the greatest length from the distance from $D$ to $E$. The distance $B$ to $B_{1}$ is small because the aim is to prevent tension on the tissues where $B_{1}$ and $C_{1}$ are approximated.

Although this wound closure violates one principle of oral surgical technic in that the closure of the vertical incision is made over the socket where the specimen was removed, and so is unsupported by bone, there has been no loss of flap tissue. The postoperative course for the patients has been uneventful, and there has been no reports of pain or swelling in the areas of the removed specimen that differed from that occurring in the areas of routine tooth removal.

The specimens were fixed in neutral formalin, decalcified, and embedded in celloidin. A large number of sections were prepared and stained from each block.

CLINICAL OBSERVATIONS AND CLINICAL AND HISTOLOGIC MEASUREMENTS

The healing after the gingivectomy progressed in the usual manner. The sample is inadequate for valid observations with regards to the role of presurgical inflammation and calculus or traumatic occlusion in the rate of postgingivectomy healing. However, there was no obvious indication that these factors influenced the healing as observed clinically.

The clinical and microscopic measurements have been compiled in Table 1. Presence of antagonist for the experimental tooth is marked with a plus sign $(+)$ and absence of antagonist with a negative sign (-). In no instance did the patients have occlusal support posterior to the cuspids. The measurements in the presurgical columns were obtained immediately prior to the anesthesia for the gingivectomy. The measurement from the notch to the surgical level of attachment was made after completion of the postgingivectomy planing of the root. The figures recorded in the first two "posthealing" columns were obtained immediately before the removal of the block specimen. The data in the third and fourth "posthealing" columns are from the prepared tissue sections. A calibrated eyepiece for the microscope was used, and the average of three measurements obtained from different sections of each specimen was recorded. In one instance (Case 8 ) the notch did not extend into the dentin, so no histologic measurements could be recorded. In the last three columns under "results" appears a comparison between various measurements.

The most significant and somewhat disturbing observation is the tendency to move the attachment level apically during gingivectomy. This is expressed in the difference between the measurements taken at the completion of the gingivectomy and the presurgical measurements between the notch and the attachment at the bottom of the crevice (see first column under "results," Table 1). It has been shown in previous papers that such measurements usually extend to the bottom of the epithelial attachment. ${ }^{13}$ In seven out of ten specimens the distance from the notch to the tissue attachment after the gingivectomy has increased compared with the pre- 
surgical measurements. The average loss of attachment during surgery was about one half a millimeter with the greatest loss of $1.5 \mathrm{~mm}$. in Case 6. This lowering of attachment was not a result of the gingival excision; but it happened during the root planing after the surgery. Although it can be argued that this represents a very small distance and the method of measuring easily may compound an error of this magnitude, the trend was definitely one to deepen the attachment level, as seen also in the second column under "results." Here the presurgical measurements of crevice depth have been subtracted from the posthealing measurements. Again in seven cases the crevice appeared deeper (average $0.8 \mathrm{~mm}$., maximum $1.7 \mathrm{~mm}$.) after the healing period.

The next to the last column shows the difference between the clinical measurements immediately after the root planing and the microscopic measurements between the notch and the apical marks in the cementum made during the root planing. The average discrepancy between these measurements is about one-half a millimeter, and the tendency is for the clinical measurements to be slightly greater than the microscopic. It should be realized that the clinical measurements were taken from the surface of the enamel, while the histologic measurements were obtained from the surface of the underlying dentin due to the loss of the enamel in decalcification. The former measurements represent the hypotenuse of a triangle from the notch to the periodontal attachment on the cementum, and this hypotenuse would be shorter if the measurements started at the dentin margin of the notch. The point on the root was the same in both instances. When this source of error is taken into account, there appears to be a good correlation between the clinical and the microscopic measurements since the average discrepancy is less than one-half a millimeter.

The last column of Table 1 indicates the difference between the posthealing clinical measurement from the notch to the bottom of the crevice and the microscopic measurements between the notch and the apical mark in the cementum from the root planing. These measurements show a fine correlation with an average difference of only $0.3 \mathrm{~mm}$. with the tendency for the microscopic measurements to be slightly shorter than the clinical ones. It therefore can be assumed that clinical measurements of the type used in this study provide information with an average accuracy or error level of about $0.5 \mathrm{~mm}$.

\section{HISTOLOGIC FINDINGS}

Since the specimens were obtained at various time intervals between zero and 44 days (See Table 1) after gingivectomy and show a great deal of variation, a fairly detailed histologic description will be submitted for each case.

Case 1. The specimen was removed immediately after gingivectomy and root planing (see Fig. 3). The gingivectomy excision had passed well above the alveolar crest and left the periosteum intact. It appears that the epithelial attachment had been completely removed by the surgery. There is a shallow $(0.2 \mathrm{~mm}$.) crevice of separation between the tooth and the

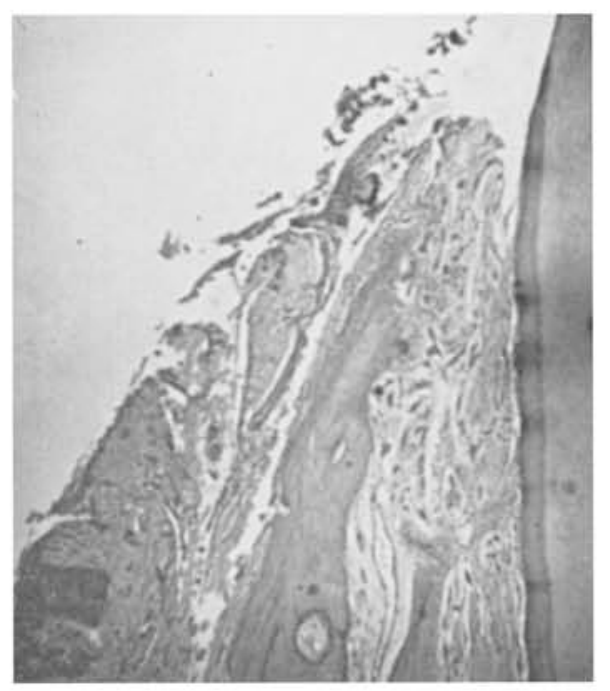

Fig. 3. Case 1. Specimen immediately after gingivectomy. Shallow crevice-like separation between the tooth and the soft tissues. (Magnification $\mathrm{X} 40$ ) 
fibrous connective tissue in the area of indentation from curettement of the root surface. This is the only specimen where paraffin embedding was used instead of celloidin, and this resulted in some artifacts.

Case 2. This specimen represents a twoday followup of gingivectomy (see Fig. $4 \mathrm{~A}$ and $4 \mathrm{~B}$ ). The wound is covered by coagulated blood with visible fibrin strands and a number of polymorphonuclear white blood cells. There is evidence of necrosis at the margin of the wound. Although the soft tissues are closely adapted to the surface of the cementum, there is evidence of a surgical split between the tooth and the soft tissues of about one-half millimeter in depth. In this area of severance there is evidence also of instrumentation on the cemental surface. The epithelial attachment is completely removed. No indication of healing can be seen in the connective tissue. However, at the border of the wound there are indications of beginning epithelial regeneration. Mild chronic inflammation can be observed in the immediate vicinity of the wound and around some blood vessels on the labial surface of the alveolar crest. Areas of previous cemental resorption slightly apical to the crest are undergoing repair, probably associated with loss of opposing teeth prior to removal of the specimen.

Case 3. Four-day followup of gingivectomy (see Fig. 5). No remains of the epithelial attachment are present. There is evi-

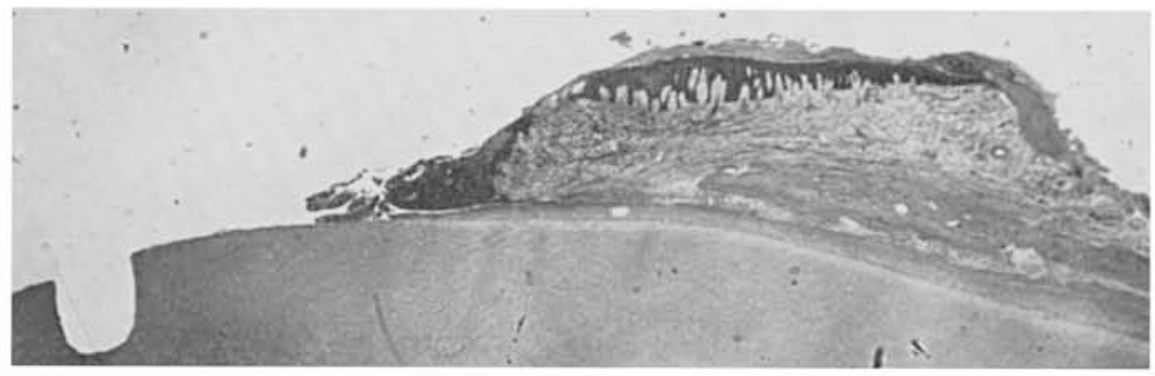

Fig. 4A. Case 2. Two days after gingivectomy. Section from a typical specimen with the coronal noteh extending into dentin. Coagulated blood on top of the gingivectomy wound where the postsurgical dressing did not fit aceurately. (Magnification X9)

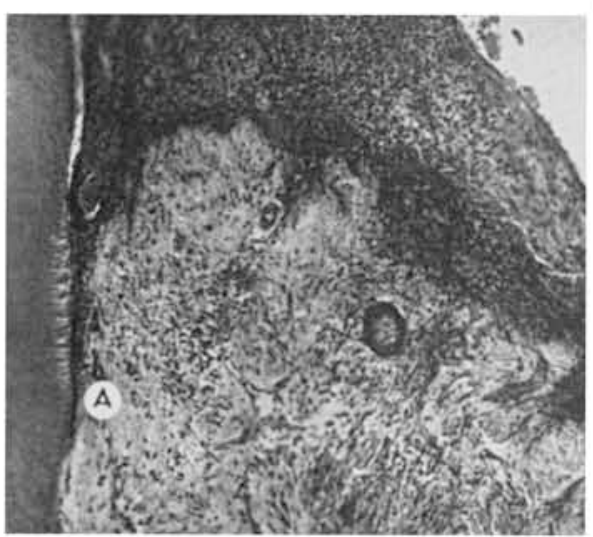

Fig. 4B. High magnification of the gingivectomy wound seen in Fig. 4A. Necrosis at the wound margin. Demareation of root planing at "A". (Magnification X133)

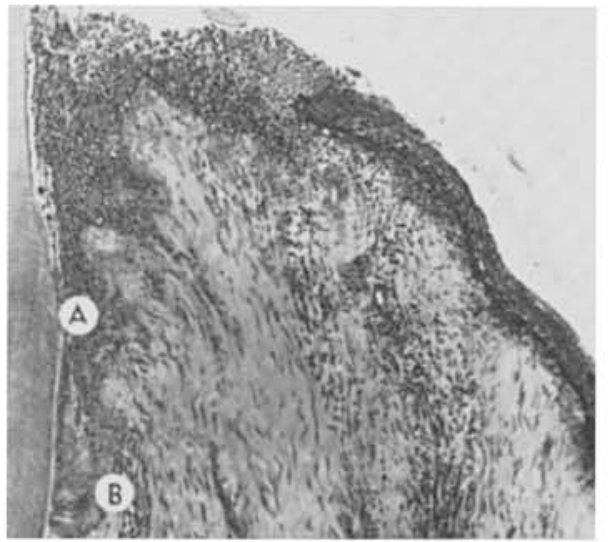

Fig. 5. Case 3. Four days after gingivectomy. Demarcation of root planing at ". $\mathrm{A}$." Boundary of soft tissue separation from the tooth at "B" More than one-half of the gingivectomy wound is covered by new epithelium. (Magnification
X133) 
dence of partial epithelization of the wound, but the epithelium has not reached the surface of the tooth. The epithelium is growing under the inflammatory exudate on the wound surface. This exudate is made up mainly of polymorphonuclear cells. There is evidence that the instrumentation during the root planing extended slightly apically to the crest of the soft tissues. The crevicular tear of the soft tissues extends slightly apically to the mark on the root surface from the scaling. Inflammatory exudate also is present in this surgical crevice. There is almost no connective tissue or endothelial proliferation. Mild inflammation is seen at the borders of the wound. The periodontal membrane is normal and there is evidence of new bone formation at the alveolar crest. This tooth did not have opposing teeth and was probably erupting.

Case 4. Six-day followup of gingivectomy (see Figs. 6A and 6B). There is complete epithelization of the gingival wound and a new epithelial attachment to the cementum has formed almost at the cementum-enamel junction. This new epithelial attachment extends slightly beyond the demarcation of the curettement on the root surface $(0.1 \mathrm{~mm}$.). The combined depth of the gingival crevice and the epithelial attachment is only about $0.2 \mathrm{~mm}$. There is a small amount of granulation tissue in the vicinity of the previous wound adjacent to the regenerated epithelium. Mild chronic inflammation can be observed under the surface of the new epithelium. A few lymphocytes and plasma cells are located in interstitial tissue spaces extending to the alveolar crest. However, there is no evidence of crestal resorption.

Case 5. Seven-day followup after gingivectomy (see Fig. 7A). The regenerated epithelium covers the gingival surface completely. There is no keratinization, but some rete peg formation. The new epithelium is also growing down along the root surface in the area of the surgical separation at the time of the root planing. One deep rete peg also is running parallel to the developing very thin new epithelial attachment. In

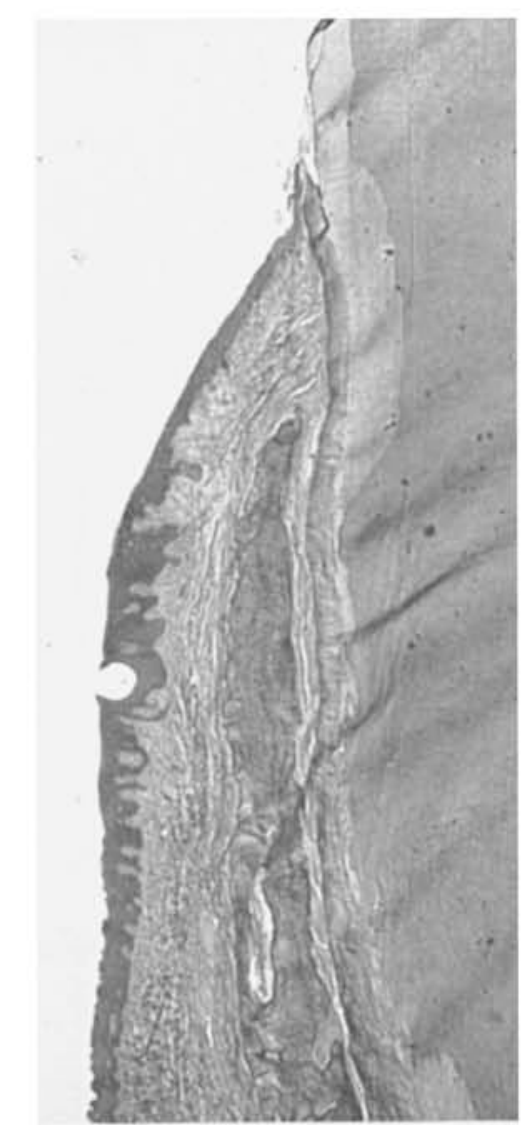

Fig. 6A. Case 4. Six days after gingirivectomy wound. (Mamnification X17)

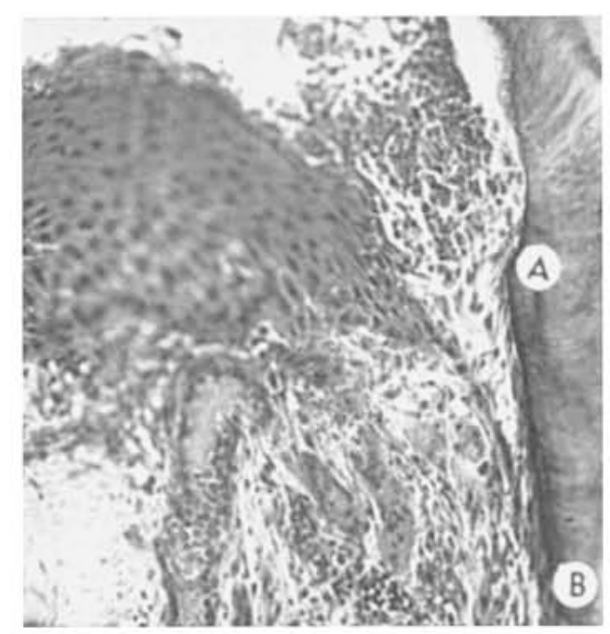

Fig. 6B. High magnitication from Fig. 6A. Demarcation of root planing at "A". Bottom of new epithelial attachment at "B". (Magnification Х276) 


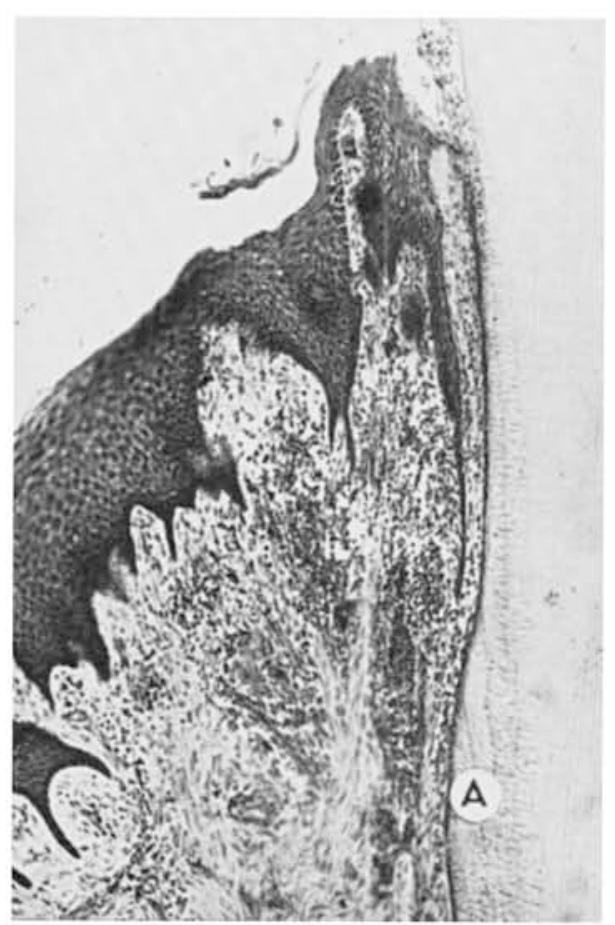

Fig. 7A. Case 5. Seven days after gingivectomy. Demareation of root planing at "A". Close adap tation and partial epithelization at the junction of the soft tissues and the tooth above " $A$ ".

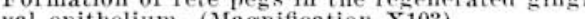
val epithelium. (Magnification X103)

some sections the new epithelial attachment has reached the apical boundary of the cemental curettement; in other sections (see Fig. 7A) there is only partial epithelization of the crevice created by the root planing. A slight amount of newly formed delicate granulation tissue can be seen on the wound surface against the cementum, but there is no indication of a new connective tissue attachment to the root in these areas, and no cementoid can be found. Mild chronic inflammation extends into the periodontal membrane (see Fig. 7B), but in spite of the inflammation there is indication of new bone formation at the alveolar crest. This patient had only anterior maxillary teeth against lower teeth. The tooth included in the specimen has mobility \#1 both prior to the gingivectomy and at the time the specimen was removed. It appears that the new $0.8 \mathrm{~mm}$. deep gingival crevice or epithelial attachment in this specimen may be the

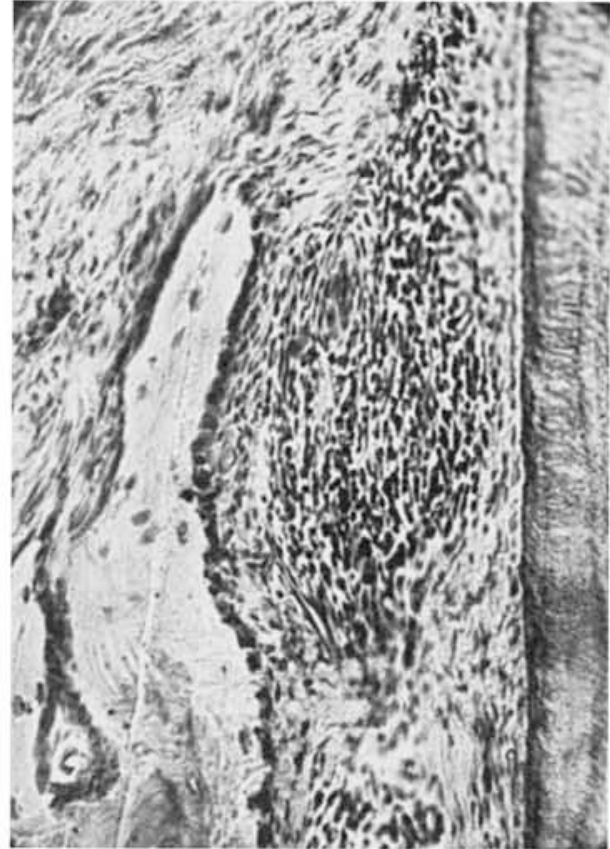

Fig. 71. From same specimen as Fig. 7A. Chronic inflammation in periodontal membrane. New bone formation at alveolar crest. (Magnification $\times 230$ )

result of careless root planing associated with the gingivectomy.

Case 6. Seven-day followup after gingivectomy (see Fig. 8). The gingivectomy wound is epithelized in part, and in most sections the regenerating epithelium has almost reached the surface of the tooth. In no instance is contact established between the new epithelium and the tooth. There are no remains of the presurgical epithelial attachment present. Some sections show newly formed granulation tissue on the surface of the old wound. In other sections (see Fig. 8) the connective tissue appears degenerated from the surgical trauma. In these latter areas there is no granulation tissue and slow epithelization. There has been some surgical separation established between the tooth and the soft tissues at the time of the removal of the specimen. Mild chronic inflammation is seen in the supracrestal tissues and severe chronic inflammation in the periodontal membrane. However, there is no evidence of bone re- 
sorprion at the alveolar crest. This tooth was in traumatic occlusion. It had mobility \#1 before the gingivectomy and mobility \#2 at the time the specimen was removed.

Case 7. Seven-day followup after gingivectomy. This lower incisor had severe periodontitis with a large amount of supraand subgingival calculus. The gingivectomy was done without presurgical scaling. The patient had a deep overbite, and the tooth had mobility \#1. There is partial epithelization of the wound surface (see Fig. 9). However, fibrin and exudate covers about half of the wound. The gingiva above the demarcation on the root from the scaling and root planing is made up of highly inflamed granulation tissue. The scaling at the time of the gingivectomy had extended almost to the alveolar crest. There are no remains of the old epithelial attachment. Particles of cementum and other nonidentifiable calcified material (probably calculus) are seen within the gingival granulation tissue. Severe inflammation extends

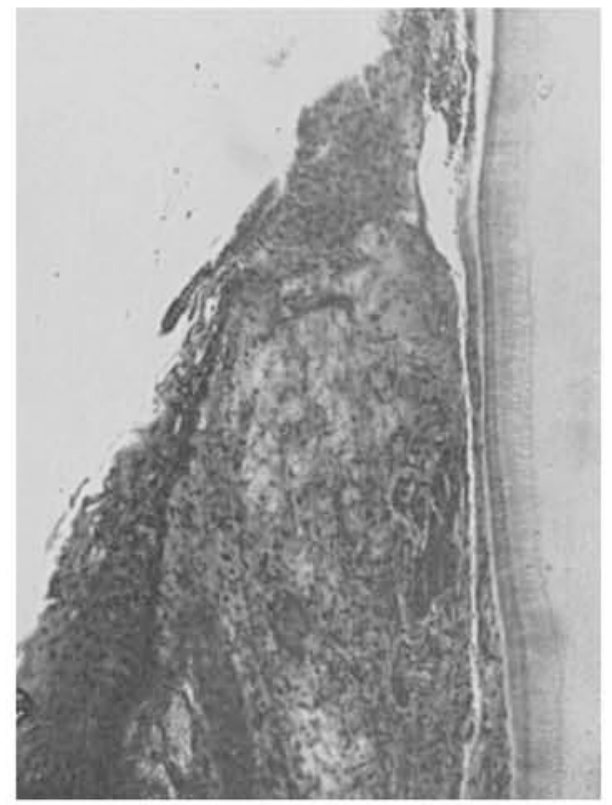

Fig. 8. Case 6. Seven days after gingivectomy. The gingivectomy wound is not completely epithelized. Necrosis and degeneration of the connective tissue under the wound surface. Debris on top of the wound. Surgical separation between tooth and soft tissues. (Magnification x133) both into the periodontal membrane and down on the labial aspect of the alveolar process. Active bone resorption is seen at the alveolar crest and on the labial surface of the alveolar process. However, there is no indication of resorption of the alveolar bone from the periodontal membrane.

Case 8. Twelve-day followup after gingivectomy. The gingival surface is completely epithelized and keratinization has started (Figs. 10A and 10B). Some sections show complete new epithelial attachment to the demarcation of the root planing. This new epithelial attachment is only two to three cell layers thick. In other sections (Fig. 10B) only one-half of the area of the separation between the soft tissues and the tooth has been covered by epithelium. There is no evidence of cementoblastic activity in the area of separation, and subsequent adaptation of connective tissue to the tooth. There is mild diffuse chronic inflammation in the entire gingiva, and

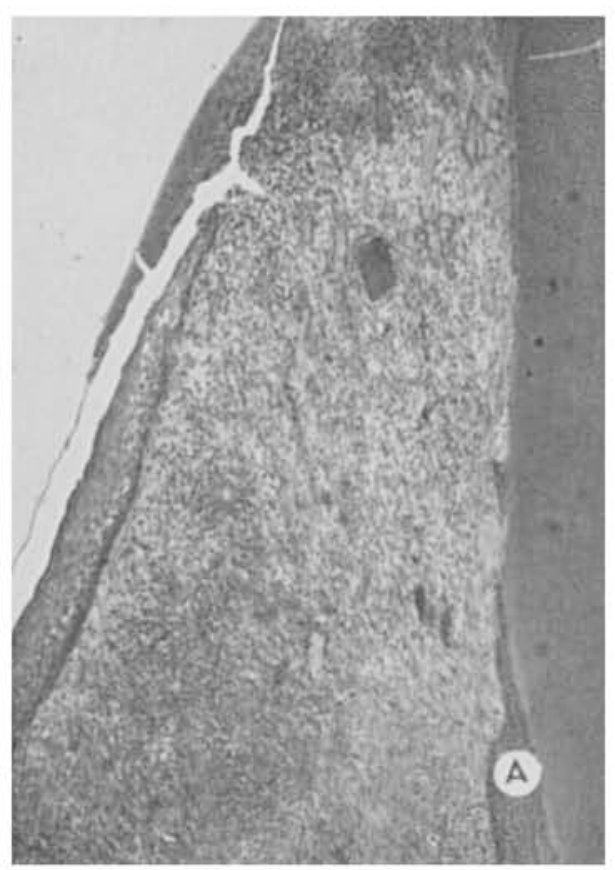

Fig. 9. Case 7. Seven days after gingivectomy. Partial epithelization of the wound. The epithelium is growing under the remains of the blood clot and debris on the wound surface. End of root planing at "A". Close adaptation of granulation tissue to the tooth surface (Magnification X87) 


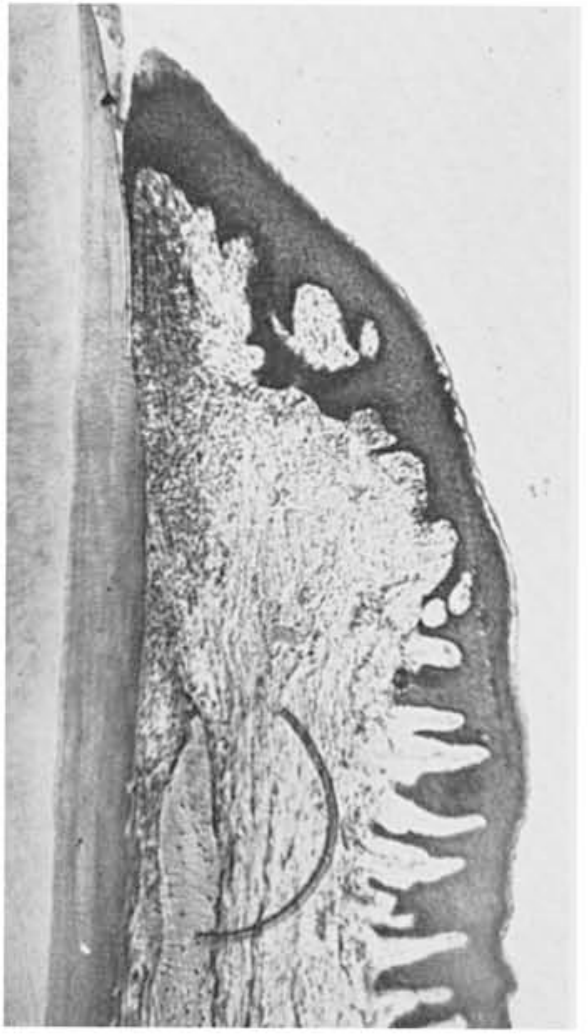

Fig. 10A. Case 8. Twelve days after gingivectomy. Complete epithelization and beginnin keratinization of gingiva. Inflammation extend tion $\mathrm{X} 55$ )

some spread of inflammation into the periodontal membrane. There is active resorption of the alveolar crest from the periodontal membrane side (see Fig. 10C). The tooth was without antagonist the last five days prior to the removal of this specimen.

Case 9. Twelve-day followup after gingivectomy. The labial surface of the gingiva is completely epithelized (see Fig. 11). There is evidence of curettement on the root surface extending $0.6 \mathrm{~mm}$. apically to the new free gingival margin. The crevicular epithelization or new epithelial attachment extends about to the middle of this potential crevice. Between the end of the new epithelial attachment and the demarcation for the root planing, the connective tissue is closely adapted to the tooth. Fibroblasts are in very close proximity to the

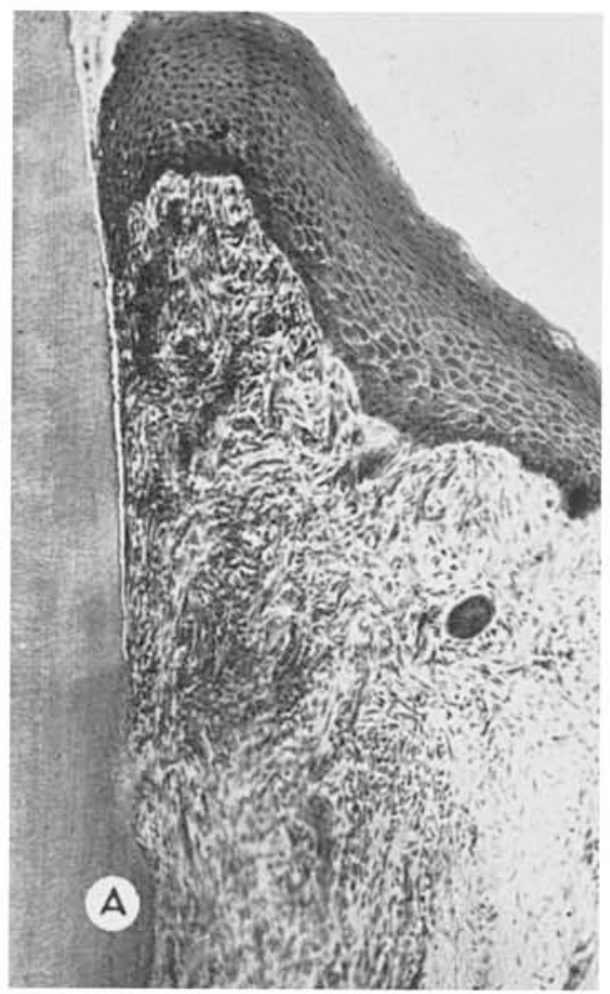

Fig. 10B. High magnification from Fig. 10A. Demarcation of root planing at "A". Less than one-half of the distance from the gingival marreattachment. (Magnification X108)

cementum with no visible intercellular substance between the cells and the tooth. There is a small amount of granulation tissue, but no indication of cementoid or new connective tissue attachment in this area. In some sections a split appeared indicating an open crevice (see Fig. 11); however, this is an artifact that most of the sections did not show. Chronic inflammation extends from the gingiva and into the periodontal membrane. There is evidence of bone resorption on the periodontal membrane side of the alveolar crest and alveolar bone. New cementum is being formed on the root surface apically to the alveolar crest. The tooth did not have opposing teeth. The mobility was \#1. The gingivectomy dressing had been removed four days prior to removal of the specimen.

Case 10. Forty-four-day followup after 
gingivectomy. The healing has been complete with an epithelized gingival crevice and epithelial attachment extending 0.1 $\mathrm{mm}$. apically to the demarcation for the root planing (see Fig. 12A). New calculus and bacterial plaques have been attached to the cemental surface which in some sections appears very rough after the root planing (see Fig. 12B). There is severe chronic gingivitis with small areas of ulceration within the gingival crevice. The inflammation extends to the alveolar crest, and in some sections there is evidence of the inflammation extending into the periodontal membrane. At the bottom of the epithelial attachment there is inflammation and proliferative activity of the epithelium (see Fig. 12C), with the characteristic budding of the epithelium away from the root surface often seen in active periodontitis.

Areas of previous resorption of cementum apically to the alveolar crest have been repaired and new bone has formed on both the alveolar crest and on the periodontal membrane side of the alveolar bone. The tooth was without antagonists. The depth of the gingival crevice clinically was 1.6 $\mathrm{mm}$.; microscopically $1.2 \mathrm{~mm}$. (see Table 1).

EVALUATION AND CLINICAL

SIGNIFICANCE OF FINDINGS

The most important observation in this study was the tendency to sever connective tissue attachment during scaling and root planing after the gingivectomy excision. A similar experience has been reported by Waerhaug ${ }^{6}$ in dogs. This came as an unpleasant observation, since we liked to think that we were careful in the performance of the operation.

The extent of final healing following such separation cannot be completely assessed from this study, but the tendency appeared to be for the epithelial attachment to grow down to the demarcation from instrumentation on the cemental surface. A "creeping reattachment" in such an area of a new epithelial attachment may be pos-

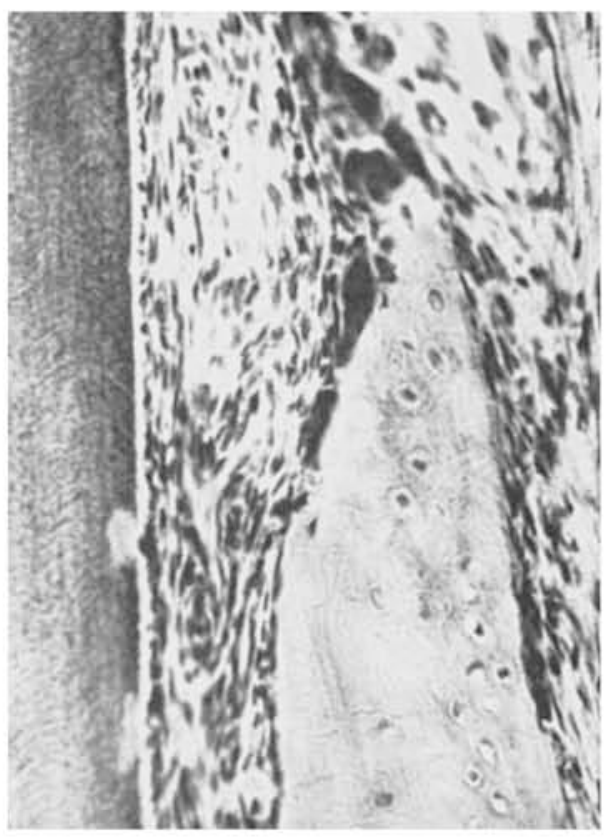

Fig. 10C. High magnification from Fig. 10A. Osteoclastic activity at the alveolar erest on the periodontal membrane side. Mild chronic inflammation. (Magnification X288)

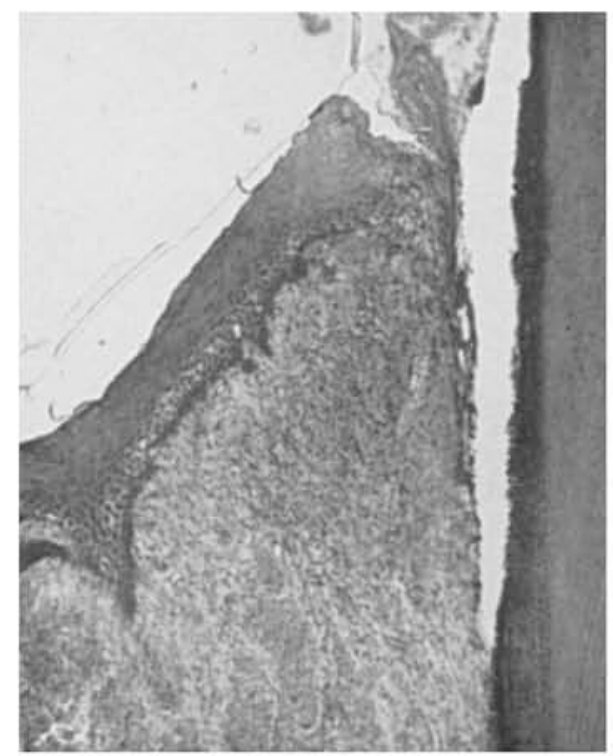

Fig. 11. Case 9. Twelve days after gingivectomy. Complete epithelization of the gingiva. The epithelium extends to the middle of the erevice. Demarcation of root planing at the bottom of the crevice. (Magnification X100) 


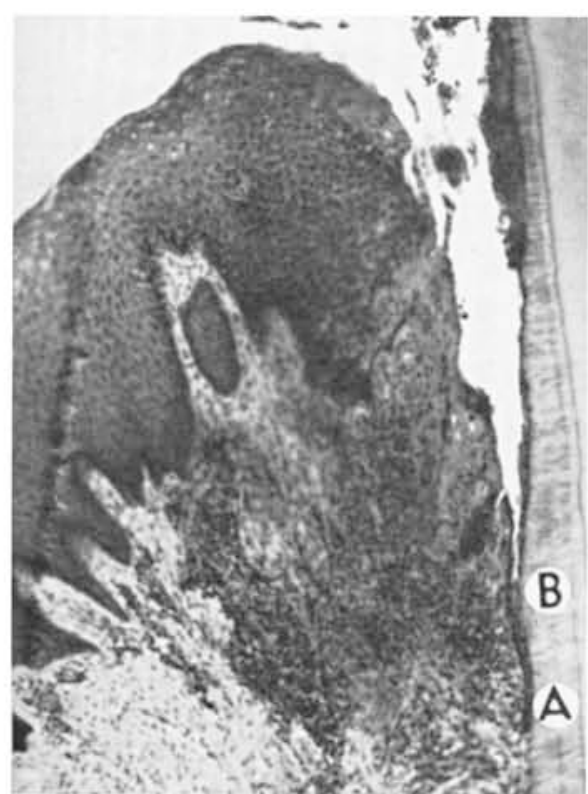

Fig. 12A. Case 10. Forty-four days after gingivectomy. The epithelial attachment extends slightly apically to " $\mathrm{A} "$. The demarcation of
the root planing is at " $\mathrm{B}$ ". (Magnification X108)

sible, but certainly it is not the common occurrence. One therefore should be extremely careful with the instrumentation on the root surface in conjunction with the gingivectomy. Since close adaptation is important for healing of such detachment, it is important that the soft tissues are immobilized against the tooth surface with suitable postsurgical dressings. A separation without curettement of the cemental surface heals much better than a separation followed by such curettement. Therefore, curettes should be used with extreme care at the junction of the soft tissues and the tooth.

The old epithelial attachment was completely removed in every case by this gingivectomy which extended to the bottom of the gingival crevice. A similar observation was made by Waerhaug ${ }^{6}$ in dogs. The new gingival crevice after a healed gingivectomy may be either the result of downgrowth of the new epithelial attachment in the area of separation between the tooth and the soft tissue, or as the result of proliferation of a new free gingiva. A combi-

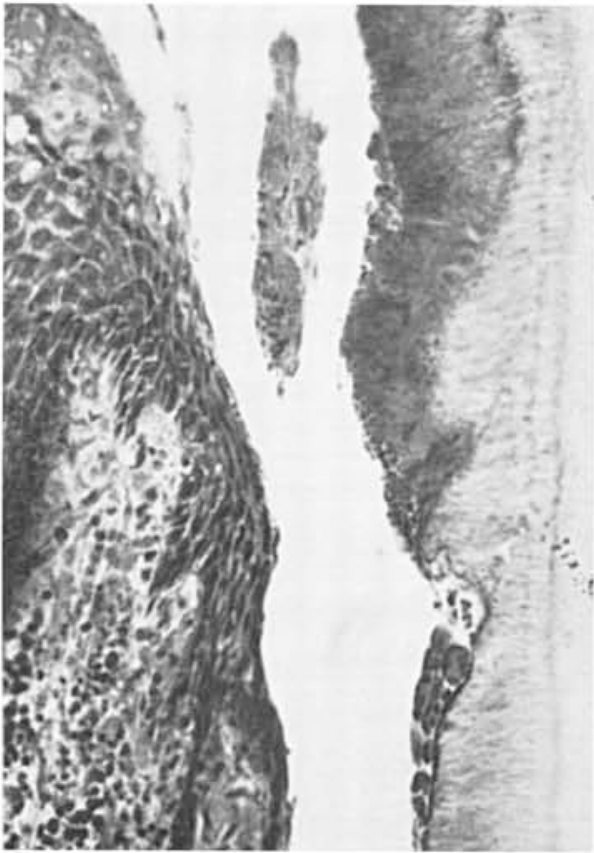

Fig. 12B. From case 10. Note rough tooth surface with adherent plaque and calculus. (Magnification $\mathrm{X} 276$ )

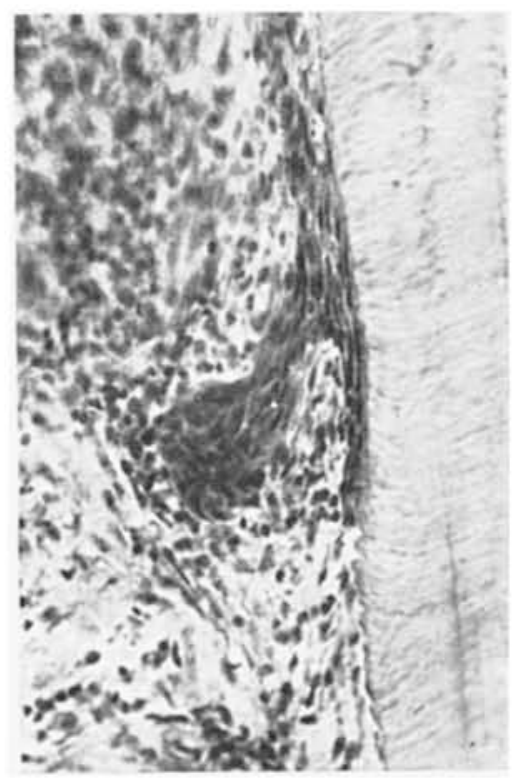

Fig. 12C. High magnification from Fig. 12A. Proliferation at the bottom of the epithelial attachment. (Magnification $\mathrm{X}^{276}$ 
nation of the two processes is probably most common.

The observations made in this investigation seem to go in favor of pregingivectomy scaling and a healing period before the gingivectomy. Soft, fragile, inflamed connective tissue at the bottom of the pocket offers much less resistance to a root planing instrument than firmly attached collagenous fibers. It also was noted in Case 7 that debris or calculus may be incorporated in the postsurgical granulation tissue and result in severe inflammation. This patient had extremely hard supra- and subgingival calculus with marked gingival inflammation at the time of the gingivectomy.

Surgical trauma from poor instrumentation may cause necrobiotic or degenerative changes in the tissues adjacent to the surgery and thereby delay and disturb the healing as seen in Fig. 8.

There was no direct effect of this simple gingivectomy upon the alveolar crest. However, bone resorption of the crest and the alveolar structures, both from the labial and from the periodontal membrane side, was observed associated with spread of inflammation from the wound and possibly from presurgical inflammation associated with the periodontal disease.

The time required for complete healing to occur after gingivectomy varied, and it is related to so many variable and partially unpredictable factors that more investigation would be needed before a meaningful report could be made. Specimens of interproximal areas would also be required for such a study.

Extension of inflammation into the periodontal membrane was observed associated with both traumatic occlusion and nonfunction, so it did not appear that the traumatic occlusion was an important factor in this unconventional spread of the gingival inflammation.

Fig. 12B illustrates well the plaque and calculus attachment to the rough cemental surface and serves to emphasize the importance of a smooth root surface following the gingivectomy. Notching of the tooth surface may also be the result of careless handling of a gingivectomy knife as seen in Fig. 13.

Case 10 also is an illustration of the well known fact that gingivectomy does not cure periodontal disease if the teeth are not kept clean. Forty-four days after the gingivectomy the epithelial attachment is migrating apically (see Fig. 12C) as part of active destructive periodontal disease.

The only practical way to get data from a significant number of patients following periodontal therapy over a long period of time is by clinical investigation. The comparison between the clinical and histomicroscopical measurements in this and previously reported studies ${ }^{13}$ indicates that clinical assessments and measurements of the connective tissue attachment level on the tooth are very reliable within a rather insignificant level of error providing that a suitable and careful examination technic is used.

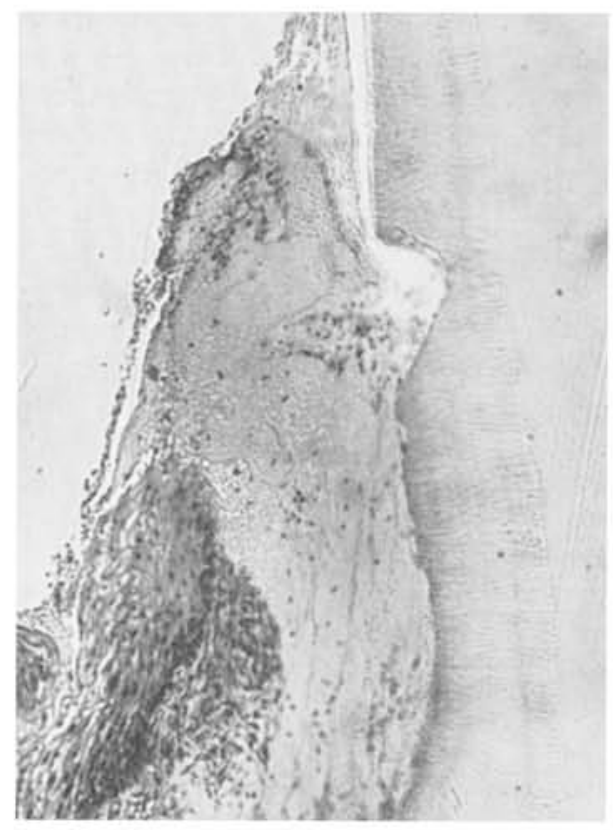

Fig. 13. Notch in cementum from the gingivectomy knife (Bard-Parker \#12B). (Magnification 


\section{SUMMARY}

1. The clinical and histologic sequelae to simple gingivectomy have been studied in nine patients, providing ten postgingivectomy specimens of teeth and surrounding tissues.

2. There was an average severance of connective tissue attachment of about 0.5 $\mathrm{mm}$. during the root planing part of the gingivectomy.

3. The epithelial attachment was removed completely in all instances by simple gingivectomy to the bottom of the clinical gingival crevice.

4. The new gingival sulcus following gingivectomy may be the result of either epithelium growing down into the crevice made between the tooth and the soft tissues during root planing, or connective tissue proliferation building up a new free gingiva. Usually it is a combination of these two mechanisms.

5. Cemental curettement in most instances was followed by a downgrowth of the new epithelial attachment instead of connective tissue reattachment.

6. No specific correlation was found between traumatic occlusion and spread of gingival inflammation into the periodontal membrane.

7. Good correlation was observed between the clinical and the histomicroscopical measurements.

\section{CONCLUSTONS}

1. Extreme care should be observed when the teeth are scaled and planed in association with gingivectomy in order to avoid permanent loss of periodontal attachment.

2. The tendency is to lose some attachment for a tooth each time a gingivectomy is done.

3. A simple gingivectomy or gingivoplasty should not be extended beyond the bottom of the clinically measurable crevice unless this is needed for elimination of pockets and establishment of proper contour.

4. Poor surgical technic delays and interferes with the healing.

5. Clinical measurements can provide reliable information about the connective tissue attachment level of teeth.

\section{REFERENCES}

1. Mann, J. B., and Kaplan, Harry. Histologic studies of block sections of teeth and investing structures removed at intervals following surgical operation. (Abstract) J. Dent. Res., 20:281. June 1941.

2. Orban, Balint, and Archer, E. A. Dynamics of wound healing following elimination of gingival pockets. J. Orthodont. and Oral Surg., 31:40-54, Jan. 1945 .

3. Bernier, J. L., and Kaplan, Harry. The repair of gingival tissues after surgical intervention. J. Am. Dent. A., 35:697-705, Nov. 15, 1947.

4. Ambrose, J. A., and Detamore, R. J. Correlation of histologic and clinical findings in periodontal treatment. J. Periodont., 31:238-242, July 1960.

5. Glickman, Irving. The effect of prescaling upon healing following periodontal surgery-a clinical and histologic study. J. Dent. Med., 16:19-22, Jan. 1961.

6. Waerhaug, Jens. Depth of incision in gingivectomy. Oral Surg., Oral Med., and Oral Path., $8: 707-18$, July 195s.

7. Waerhaug, Jens, and Löe, Harald. Tissue reaction to gingivectomy pack. Oral Surg., Oral Med., and Oral Path., 10:923-37, Sept. 1957.

8. McHugh, W. D., and Persson, P. A. Fluorescence microscopy of healing gingival epithelium. Acta Odont. Scand., 16:205-18, Aug. 1958.

9. Persson, P. A. The healing process in the marginal periodontium after gingivectomy, with special regard to the regeneration of epithelium. Odont. Tskr., 67:593-615, Nov. 1959.

10. Stahl, S. S. Response of the periodontium, pulp, and salivary glands to gingival and tooth injury in young adult male rats. I. Periodontal tissues. Oral Surg., Oral Med., and Oral Path., 13:613-26, May 1960.

11. Stahl, S. S. Healing gingival injury in normal and systemically stressed young adult male rats. J. Periodont., 32:63-73, Jan. 1961.

12. Stahl, S. S., and Persson, P. A. Reattachment of epithelium and connective tissue following gingival injury in rats. J. Periodont., 33:51-55, Jan. 1962.

13. Kohler, C. A., and Ramfjord, S. P. Healing of gingival mucoperiosteal flaps. Oral Surg., Oral Med., and Oral Path., 13:89-103, Jan. 1960. 